\title{
Effect of Sewage Water Irrigation Frequency on Growth, Yield and Heavy Metals Accumulation of Tomato and Okra
}

\author{
Safina $\mathrm{Naz}^{1 *}$, Muhammad Akbar Anjum ${ }^{1}$, Bushra Siddique ${ }^{2}$, Syed Atif Hasan Naqvi ${ }^{*}$, Sajid Ali ${ }^{1}$, Hassan \\ Sardar ${ }^{1}$, Sakeena Tul Ain Haider ${ }^{1}$, Muhammad Asif Zulfiqar ${ }^{4}$, Hajra Azeem $^{3}$, Sibghat Ullah ${ }^{5}$, Shah Pasand ${ }^{6}$ \\ and Zehri Khan ${ }^{5}$
}

${ }^{1}$ Department of Horticulture, Bahauddin Zakariya University, Multan, Pakistan; ${ }^{2}$ Department of Entomology, The Islamia University of Bahawalpur, Pakistan; ${ }^{3}$ Department of Plant Pathology, Bahauddin Zakariya University, Multan, Pakistan; ${ }^{4}$ PARC, Research and Training Station, Bahauddin Zakariya University, Multan, Pakistan; ${ }^{5}$ Pakistan Agricultural Research Council, Horticulture Research Institute, Khuzdar, Pakistan; ${ }^{6}$ Pakistan Agricultural Research Council, Social Sciences Research Institute, Quetta, Pakistan.

\begin{abstract}
The current study was aimed to inspect the efficiency of different sewage water irrigation frequency treatments on growth, yield and heavy metals accumulation of tomato and okra during 2012 and 2013. A statistical randomized complete block design was applied in the field to perform experiments having three replicates while 03 sewage water irrigation frequency treatments i.e., 5, 10 and 15-days interval. The tomato yield, their growth along with the production of the plant biomass were increased considerably with more repetitive (interval of five days) application of the sewage water. While reasonably less repetitive application (interval of fifteen days) of sewage water cause the less production of biomass, yield and growth. Moderately less repetitive applications (interval of ten days) of sewage water showed a significant increase in the addressed attributes of production in okra. The yield, as well as growth aspects of the okra, were reduced noticeably by using the reasonably less repetitive application. Frequent sewage water application resulted in an increased amount of $\mathrm{Fe}, \mathrm{Cu}, \mathrm{Pb}, \mathrm{Cr}, \mathrm{Ni}$ and $\mathrm{Cd}$ in okra pods and tomato fruits than moderately and less repetitive irrigation. The leaves and root of okra and tomato displayed the higher contents of metal when these crops were cultivated with a repetitive application of the sewage water. The final result revealed that the metal concentration high in tomato and okra plant parts with decreasing irrigation intervals of sewage water.

Received | July 20, 2020; Accepted | August 28, 2020; Published | October 09, 2020

*Correspondence | Syed Atif Hasan Naqvi and Safina Naz, Department of Horticulture, Bahauddin Zakariya University, Multan; Department of Plant Pathology, Bahauddin Zakariya University, Multan, Pakistan; Email: atifhasanshah@hotmail.com, safinnaz@bzu.edu.pk

Citation $\mid$ Naz, S., M.A. Anjum, B. Siddique, S.A.H. Naqvi, S. Ali, H. Sardar, S.T.A. Haider, M.A. Zulfiqar,H. Azeem, S. Ullah, S. Pasand and Z. Khan. 2020. Effect of sewage water irrigation frequency on growth, yield and heavy metals accumulation of tomato and okra. Pakistan Journal of Agricultural Research, 33(4): 798-809.

DOI | http://dx.doi.org/10.17582/journal.pjar/2020/33.4.798.809

Keywords | Irrigation frequency, Sewage water, Growth, Yield, Heavy metals, Vegetables
\end{abstract}

\section{Introduction}

W ater is necessary for the physiological activity of crop plants. Too much irrigation at any stage can delay maturity and harvesting and promote vegetative growth, whereas inadequate and less irrigation cause water stress and reduce quality and yield (Yazgan et al., 2008). Proper irrigation time and interval is necessary for production of good quality vegetables. Water shortage at the early stage of growth and development usually results in reduced yield. If the water shortages occur later in the growing season, it may not only adversely affect the quality of vegetables but also reduce the total yield. 
Large sized fruits and greater yields were obtained when tomato plants were irrigated at 10 days interval compared with other irrigated intervals (Mani et al., 2007). However, Gudugi et al. (2012) recorded a higher number of tomato fruits and greater yield when plants were irrigated at 4 days interval than with 8 days interval. Okra is a warm-season crop and has considerable resistance to drought. Water shortage affects the morphological and physiological processes of the plants, which are associated with growth and development (Moniruzzaman et al., 2007; Yadav and Dhankhar, 1999). Drought stress affects photosynthesis, respiration, nutrient metabolism, and membrane stability index, which ultimately reduces plant growth and productivity of plants (Naveed et al., 2009). Maturity and harvesting of crop were delayed due to the excessive irrigations or shorter irrigation intervals, whereas the yield and quality of crop were also reduced due to insufficient irrigation (Ramalan and Nwokeocha, 2000; Sankar et al., 2008). Greater pod length, pod weight and yield of okra genotypes were recorded under 10 days irrigation interval (Khalil, 2004).

Use of sewage water has positive effects on plant biomass, plant height and yield of tomatoes enriched with vital plants macro and micronutrients (Khan et al., 2011). Similar results have been reported in okra by Khalil (2004) and Rehman (2007). Keeping in view of these facts, the present study was envisaged to determine the effect of frequency of irrigation with sewage water on growth, yield and heavy metals concentration in tomato and okra.

\section{Materials and Methods}

Six weeks old tomato seedlings were transplanted on both sides of $1 \mathrm{~m}$ wide beds with a plant to plant distance of $20 \mathrm{~cm}$ on $10^{\text {th }}$ February 2012 ( $1^{\text {st }}$ year crop) and $10^{\text {th }}$ February 2013 ( $2^{\text {nd }}$ year crop). By using both margins/border of ridges, okra seeds were sown at $60 \mathrm{~cm}$ distance, having PXP distance $20 \mathrm{~cm}$ on $5^{\text {th }}$ March 2012 ( $1^{\text {st }}$ year crop) and $5^{\text {th }}$ March 2013 ( $2^{\text {nd }}$ year crop). All the cultural practices were carried out according to the requirements of crops and experiments.

The crops were irrigated with sewage water at different intervals/ frequencies. By using these 03 treatments of application (frequencies of sewage water application) via more repetitive application i.e. 5 days interval, moderately less repetitive i.e. 10 days interval and less repititive i.e. 15 days interval, which were replicated thrice in a randomized complete block design (RCBD). The data were collected on different growth and yield parameters and heavy metals accumulation in different parts of these vegetables.

\section{Determination of heavy metals}

Pre-treatment and chemicals: The vegetable samples were washed with distilled water to remove any dirt or soil particles and air-dried at room temperature for sixty minutes. After that, sliced the samples into small pieces and incubated in the oven for 72 hours at $70{ }^{\circ} \mathrm{C}$. Crushed the samples into chilled mortar and pestle until the fine powdered is prepared. The prepared fine powder filter through a muslin cloth and preserved in disinfected polythene bags. Required chemicals and heavy metals standards (1ml) viz., $\mathrm{Cr} \mathrm{Pb}, \mathrm{Ni}, \mathrm{Cu}, \mathrm{Cd}$, and Fe were graciously achieved from the Merck.

\section{Acid digestion and statistical analysis}

Heavy metals were extracted by digesting $500 \mathrm{mg}$ dehydrated sample from recommended treatments in $15 \mathrm{~mL} \mathrm{HNO}_{3}$ along with $5 \mathrm{ml} \mathrm{HClO}_{4}$ mixture (3:1) by using a hot plate (Velp, Italy) with the temperature at $80^{\circ} \mathrm{C}$ until the clear solutions were achieved. The Whatman No. 42 filter paper was used to filter the obtained solution in $25 \mathrm{~mL}$ DEPC water (Farooq et al., 2008). These heavy metals contents $(\mathrm{Pb}, \mathrm{Cu}, \mathrm{Ni}, \mathrm{Cd}, \mathrm{Cr}$ and $\mathrm{Fe})$ were determined in the obtained solution by utilizing the atomic absorption spectrophotometer (Thermo Scientific 3000 series, USA). The data collected were subjected to analysis of variance (ANOVA). Least significant difference test was employed to determine significant differences among the treatment means at $5 \%$ level of probability. The data were analyzed using statistical software statistix 8.1.

\section{Results and Discussion}

\section{Effect of sewage water irrigation frequency on plant growth}

Significantly greater height, relative leaf chlorophyll content and leaf area of tomato were recorded when plants were grown with frequent irrigation with the sewage water. Whereas, significantly lower values of these parameters were recorded under less frequent irrigation treatment (Table 1). The mean values for the interaction between various irrigation frequency treatments and years exhibited a statistically 
significant effect on plant height and leaf area. The taller plants were obtained with the interactive effect of frequent irrigation $\times$ year 2012, followed by the interaction of frequent irrigation $\times$ year 2013. These two treatments were statistically at par. The combined effect of less frequent irrigation $\times$ year 2013 resulted in significantly shorter plants. The combined effect of the frequent $\times$ year 2012, the frequent $\times$ year 2013 and moderately frequent irrigation $\times$ year 2013 resulted in greater leaf area. These three treatment combinations stood statistically at par with each other. Leaf area was significantly lower in the plants grown under less frequent $\times$ year 2013 (Table 1$)$.

The maximum plant height and leaf area were produced in okra grown with moderately frequent irrigation treatment, which was followed by frequent irrigation treatment. The minimum values for these parameters were obtained when plants were irrigated with less frequent irrigation treatment. All the treatments were significantly different from each other. Frequent and moderately frequent irrigation treatments were statistically similar with greater relative leaf chlorophyll content. Whereas, significantly lesser content was recorded with less frequent irrigation treatment (Table 1). Significantly higher relative leaf chlorophyll content was recorded when okra plants were grown under the frequent irrigation treatment during the year 2013 and moderately frequent irrigation treatment during the year 2012, which were statistically alike. Whereas relative leaf chlorophyll content was significantly decreased when the plants were grown under less frequent irrigation treatment during year 2013.

\section{Effect of sewage water irrigation frequency on fruiting}

The maximum number of tomato fruits, fruit length and fruit diameter were recorded in the plants which received frequent irrigation, while the minimum values for these parameters were recorded with less frequent irrigation with the sewage water. All these three irrigation frequency treatments were statistically different from each other (Table 2). The significantly higher number of tomato fruits was obtained with the interactive effect of frequent irrigation $\times$ year 2012, followed by the interaction of frequent irrigation $x$ year 2013. These two treatment combinations were statistically similar. The combined effect of less frequent irrigation $\times$ year 2013 and less frequent irrigation $\times$ year 2012 produced the minimum number of fruits. These two treatment combinations also behaved statistically alike. However, the combined effect of irrigation frequency treatments and years for fruit length and fruit diameter indicated non-significant differences among the treatment combinations.

Pod number, pod length and diameter in okra were significantly affected by various irrigation frequency treatments with the sewage water. Significantly greater number of pods and pod diameter were recorded with moderately frequent irrigation treatment when compared with frequent and less frequent irrigation treatments. However, latter two treatments were statistically similar. The maximum pod length was observed in the plants under moderately frequent irrigation treatment, followed by in those grown with frequent irrigation treatment. Whereas less frequent irrigation treatment produced the shortest pods. All these three treatments were statistically different from each other.

\section{Effect of sewage water irrigation frequency on yield}

Significantly higher fresh and dry weights of fruit and yield per hectare of tomato were recorded with frequent irrigation treatment. The minimum fresh and dry weights of fruit and yield were observed in less frequent irrigation treatment. All these three treatments were statistically different (Table 2).

Moderately frequent irrigation treatment produced the maximum fresh weight per pod of okra, followed by frequent irrigation treatment and it was significantly reduced with less frequent irrigation treatment. However, behavior of all these three treatments was statistically different. Moderately frequent irrigation produced higher dry weight per pod, followed by frequent irrigation treatment and these two treatments were statistically similar. Significantly higher pod yield was achieved in the plants under moderately frequent irrigation treatment. The frequent and less frequent irrigation treatments behaved statistically alike with lower yields (Table 3). Fresh weight per pod was not affected by the interactive effect of irrigation frequency treatments and years. However, greater dry weight of pod was observed with the interactive effect of moderately frequent irrigation treatment $x$ year 2013, moderately frequent irrigation treatment $x$ year 2012, frequent irrigation treatment $\times$ year 2013 and frequent irrigation treatment $\times$ year 2012. All these four treatment combinations were statistically at par with each other. While the lowest values for a dry weight of pod was observed with the interactive 
Table 1: Effect of sewage water irrigation frequency on growth of tomato and okra.

Frequency of irrigation water

Plant height $(\mathrm{cm})$

Frequent

Moderately frequent

Less frequent

Mean

Relative leaf chlorophyll content

Frequent

Moderately frequent

Less frequent

Mean

Leaf area $\left(\mathrm{cm}^{2}\right)$

Frequent

Moderately frequent

Less frequent

Mean
Tomato

Year 2012

\section{Year 2013}

Okra

Year 2013

Mean

Mean

Year 2012

\begin{abstract}
$85.23 \mathrm{a}$
\end{abstract}
$84.93 a$

$85.08 \mathrm{~b}$

$91.95 \mathrm{a}$

$83.60 \mathrm{~b}$

$83.23 \mathrm{~b}$

$77.16 \mathrm{~d}$

$83.41 \mathrm{~b}$

$91.76 \mathrm{a}$

$68.40 \mathrm{a}$

$81.80 \mathrm{a}$

$69.36 \mathrm{a}$

$82.14 \mathrm{a}$

$84.78 \mathrm{a}$

$83.40 \mathrm{a}$

$63.50 \mathrm{a}$

$61.93 a$

$62.71 \mathrm{a}$

$62.70 \mathrm{~b}$

$72.0 \mathrm{a}$

$67.55 \mathrm{a}$

$55.86 \mathrm{a}$

$56.20 \mathrm{~b}$

$69.03 \mathrm{a}$

$62.03 \mathrm{~b}$

$65.53 \mathrm{a}$

$49.96 \mathrm{a}$

$49.85 \mathrm{c}$

$56.96 \mathrm{c}$

$62.90 \mathrm{a}$

$52.50 \mathrm{~d}$

$62.31 \mathrm{a}$

$56.58 \mathrm{a}$

$55.92 \mathrm{a}$

$24.50 \mathrm{a}$

$23.50 \mathrm{~b}$

$24.46 \mathrm{a}$

$24.48 \mathrm{a}$

$198.67 \mathrm{a}$

$197.80 \mathrm{a}$

$198.23 b$

23.86ab

$23.68 \mathrm{~b}$

204.77a

$203.77 \mathrm{a}$

$204.27 \mathrm{a}$

$20.63 \mathrm{~d}$

$21.01 \mathrm{c}$

$185.77 \mathrm{a}$

$196.40 \mathrm{a}$

184.33 a

$185.03 \mathrm{c}$

Treatment means sharing the different letter(s) in a group are significantly different at $p \geq 0.05$

Table 2: Effect of sewage water irrigation frequency on fruiting of tomato and okra.

\section{Frequency of irrigation water}

Number of fruit/pods per plant

\begin{tabular}{lcccccc} 
Frequency of irrigation water & \multicolumn{3}{c}{ Tomato } \\
& Year 2012 & Year 2013 & Mean & Year 2012 & $\begin{array}{r}\text { Okra } 2013 \\
\text { Mean }\end{array}$ \\
Number of fruit/pods per plant & & & & & & \\
Frequent & $26.16 \mathrm{a}$ & $26.03 \mathrm{a}$ & $26.10 \mathrm{a}$ & $22.33 \mathrm{a}$ & $22.50 \mathrm{a}$ & $22.41 \mathrm{~b}$ \\
Moderately frequent & $24.26 \mathrm{~b}$ & $23.90 \mathrm{~b}$ & $24.08 \mathrm{~b}$ & $26.33 \mathrm{a}$ & $25.66 \mathrm{a}$ & $26.00 \mathrm{a}$ \\
Less frequent & $22.53 \mathrm{c}$ & $22.00 \mathrm{c}$ & $22.26 \mathrm{c}$ & $21.66 \mathrm{a}$ & $21.33 \mathrm{a}$ & $21.50 \mathrm{~b}$ \\
Mean & $24.32 \mathrm{a}$ & $23.97 \mathrm{a}$ & & $23.44 \mathrm{a}$ & $23.16 \mathrm{a}$ & \\
Fruit/pod length (cm) & & & & & & \\
Frequent & $6.87 \mathrm{a}$ & $6.88 \mathrm{a}$ & $6.87 \mathrm{a}$ & $10.06 \mathrm{a}$ & $10.26 \mathrm{a}$ & $10.16 \mathrm{~b}$ \\
Moderately frequent & $5.91 \mathrm{a}$ & $5.86 \mathrm{a}$ & $5.89 \mathrm{~b}$ & $12.23 \mathrm{a}$ & $12.70 \mathrm{a}$ & $12.46 \mathrm{a}$ \\
Less frequent & $5.81 \mathrm{a}$ & $5.76 \mathrm{a}$ & $5.79 \mathrm{c}$ & $9.76 \mathrm{a}$ & $9.60 \mathrm{a}$ & $9.68 \mathrm{c}$ \\
Mean & $6.20 \mathrm{a}$ & $6.17 \mathrm{a}$ & & $10.68 \mathrm{a}$ & $10.85 \mathrm{a}$ & \\
Fruit/pod diameter $(\mathrm{cm})$ & & & & & & $1.44 \mathrm{a}$ \\
Frequent & $6.10 \mathrm{a}$ & $6.23 \mathrm{a}$ & $6.16 \mathrm{a}$ & $1.45 \mathrm{a}$ & $1.54 \mathrm{~b}$ \\
Moderately frequent & $5.80 \mathrm{a}$ & $5.73 \mathrm{a}$ & $5.76 \mathrm{~b}$ & $1.56 \mathrm{a}$ & $1.54 \mathrm{a}$ & $1.55 \mathrm{a}$ \\
Less frequent & $5.36 \mathrm{a}$ & $5.43 \mathrm{a}$ & $5.40 \mathrm{c}$ & $1.40 \mathrm{a}$ & $1.33 \mathrm{a}$ & $1.36 \mathrm{~b}$ \\
Mean & $5.75 \mathrm{a}$ & $5.80 \mathrm{a}$ & & $1.47 \mathrm{a}$ & $1.43 \mathrm{a}$ &
\end{tabular}

Tomato

Treatment means sharing the different letter(s) in a group are significantly different at $p \geq 0.05$

effect less frequent irrigation treatment $\times$ year 2013 . The highest yield was obtained with the combined effect of moderately frequent irrigation treatment x year 2012, which was statistically different from all other treatment combinations. The treatment combinations, less frequent irrigation treatment $x$ year 2012, less frequent irrigation treatment $\times$ year 2013, frequent irrigation treatment $\times$ year 2013 and frequent irrigation treatment $\times$ year 2012 were statistically at par with each other with significantly lower pod yield.

Effect of serwage water irrigation frequency on biomass production (on fresh and dry weight basis)

Total biomass (fresh and dry) of tomato was significantly affected by different irrigation frequency 
treatments. Greater values for these parameters were recorded in the plants which were grown with frequent irrigation application. Whereas the minimum fresh and dry biomass was observed with less frequent irrigation treatment. All three irrigation treatments were statistically different from each other. Significantly greater biomass (on fresh weight basis) was recorded with frequent irrigation treatment $x$ year 2012, followed by frequent irrigation treatment $x$ year 2013. These two treatment combinations were statistically alike. Whereas the minimum fresh total biomass was recorded with the interactive effect of less frequent treatments $\times$ year 2013, followed by less frequent irrigation treatment $\times$ year 2012. These two treatment combinations also behaved statistically alike (Table 4).

Different irrigation frequency treatments affected the total biomass (fresh and dry) of okra. Moderately frequent irrigation treatment resulted in significantly greater total fresh and dry biomass. Whereas, the minimum values for the parameters under study were recorded with less frequent irrigation treatment. These three treatments significantly differed from each other.

\section{Heavy metals contents in tomato fruits and okra pod}

Frequent irrigation application with sewage water resulted in greater $\mathrm{Pb}, \mathrm{Ni}$ and $\mathrm{Cr}$ contents in tomato fruits, followed by with moderately frequent irrigation treatment. These two irrigation frequency treatments were statistically at par. Significantly lower contents $(\mathrm{Pb}, \mathrm{Ni}$ and $\mathrm{Cr})$ were recorded in fruits when plants were grown with less frequent irrigation treatment. This irrigation frequency was statistically similar with moderately frequent irrigation application. Significantly greater $\mathrm{Cu}$ content was recorded in fruits when plants were grown with frequent irrigation application compared with moderately or less frequent irrigation application, which was statistically alike with lower $\mathrm{Cu}$ contents. Tomato fruits contained significantly greater $\mathrm{Cd}$ and $\mathrm{Fe}$ contents when the plants were grown with frequent irrigation application. Whereas significantly lower $\mathrm{Cd}$ and $\mathrm{Fe}$ accumulation in the fruits were recorded with less frequent irrigation application (Table 5).

Significantly higher $\mathrm{Pb}$ and $\mathrm{Cr}$ contents were noticed in okra pods of the plants grown with frequent irrigation application. Moderately and less frequent irrigation application resulted in lower $\mathrm{Pb}$ and $\mathrm{Cr}$ contents in okra pods. Okra pods contained higher $\mathrm{Ni}$ and $\mathrm{Cd}$ contents with frequent irrigation application, followed by moderately frequent irrigation. These two irrigation frequencies were statistically similar. Less frequent irrigation application resulted in lower $\mathrm{Ni}$ and $\mathrm{Cd}$ contents, which was also statistically at par with moderately frequent irrigation. Frequent irrigation application resulted in greater $\mathrm{Cu}$ content in okra pods, followed by those with moderately frequent irrigation application. These two irrigation frequency treatments were statistically alike. While significantly lesser $\mathrm{Cu}$ content was detected in pods when less frequent irrigation was applied to okra plants. Significantly greater Fe content was recorded in okra pods with frequent irrigation treatment. Less frequent irrigation application resulted in significantly lower Fe contents in okra pods.

\section{Heavy metals contents in tomato and okra leaves}

Among irrigation frequency treatments with sewage water, significantly higher $\mathrm{Pb}$ and $\mathrm{Fe}$ contents were recorded in leaves when tomato plants were irrigated frequently. Whereas less frequent irrigation resulted in significantly lower $\mathrm{Pb}$ and $\mathrm{Fe}$ contents. Greater $\mathrm{Ni}$ and $\mathrm{Cd}$ contents were recorded in leaves of the plants grown with frequent and moderately frequent irrigation treatment. Whereas less frequent irrigation application resulted in the minimum $\mathrm{Ni}$ and $\mathrm{Cd}$ contents in leaves. $\mathrm{Cu}$ and $\mathrm{Cr}$ substances were considerably higher in leaves of the plants grown with frequent irrigation application compared with moderately or less frequent irrigation application. The latter two irrigation treatments were statistically similar with lower $\mathrm{Cu}$ and $\mathrm{Cr}$ contents (Table 6).

Significantly higher heavy metals $(\mathrm{Pb}, \mathrm{Ni}, \mathrm{Cu}$ and $\mathrm{Cr}$ ) contents were recorded in okra leaves grown with frequent irrigation, followed by moderately frequent irrigation. These two irrigation frequencies were statistically at par. Whereas, less frequent irrigation treatment resulted in a lower value for $\mathrm{Pb}, \mathrm{Ni}, \mathrm{Cu}$ and $\mathrm{Cr}$ contents. This treatment was also statistically alike with moderately frequent irrigation treatment. Significantly greater $\mathrm{Cd}$ content was recorded with frequent irrigation compared, while moderately and less frequent irrigation treatments were statistically similar with lower $\mathrm{Cd}$ contents. Frequent and moderately frequent irrigation applications were statistically similar with greater $\mathrm{Fe}$ contents. Whereas significantly lower $\mathrm{Fe}$ content was recorded in okra leaves when plants were irrigated with less frequent sewage water. 
Table 3: Effect of serwage water irrigation frequency on yield of tomato and okra.

Frequency of irrigation water

Fresh weight of individual fruit (g)

Frequent

Moderately frequent

Less frequent

Mean

Dry weight of individual fruit (g)

Frequent

Moderately frequent

Less frequent

Mean

Fruit yield per hectare (tons)

Frequent

Moderately frequent

Less frequent

Mean

Treatment means sharing the different letter(s) in a group are significantly different at $p \geq 0.05$
Tomato

$\begin{array}{llllll}\text { Year 2012 } & \text { Year 2013 } & \text { Mean } & \text { Year 2012 } & \text { Year 2013 } & \text { Mean } \\ & & & & & \\ 95.46 \mathrm{a} & 95.83 \mathrm{a} & 95.65 \mathrm{a} & 8.96 \mathrm{a} & 9.00 \mathrm{a} & 8.98 \mathrm{~b} \\ 92.36 \mathrm{a} & 92.00 \mathrm{a} & 92.18 \mathrm{~b} & 9.23 \mathrm{a} & 9.26 \mathrm{a} & 9.25 \mathrm{a} \\ 88.36 \mathrm{a} & 88.16 \mathrm{a} & 88.26 \mathrm{c} & 8.11 \mathrm{a} & 8.10 \mathrm{a} & 8.10 \mathrm{c} \\ 92.06 \mathrm{a} & 92.00 \mathrm{a} & & 8.76 \mathrm{a} & 8.78 \mathrm{a} & \\ & & & & & \\ 7.83 \mathrm{a} & 7.90 \mathrm{a} & 7.86 \mathrm{a} & 1.79 \mathrm{a} & 1.80 \mathrm{a} & 1.79 \mathrm{a} \\ 6.80 \mathrm{a} & 6.63 \mathrm{a} & 6.71 \mathrm{~b} & 1.84 \mathrm{a} & 1.85 \mathrm{a} & 1.85 \mathrm{a} \\ 6.16 \mathrm{a} & 6.13 \mathrm{a} & 6.15 \mathrm{c} & 1.62 \mathrm{~b} & 1.42 \mathrm{c} & 1.52 \mathrm{~b} \\ 6.93 \mathrm{a} & 6.88 \mathrm{a} & & 1.75 \mathrm{a} & 1.69 \mathrm{a} & \\ & & & & & 10.69 \mathrm{~b} \\ 48.96 \mathrm{a} & 48.47 \mathrm{a} & 48.71 \mathrm{a} & 10.79 \mathrm{c} & 10.58 \mathrm{c} & 16.80 \mathrm{a} \\ 42.65 \mathrm{a} & 42.10 \mathrm{a} & 42.37 \mathrm{~b} & 17.99 \mathrm{a} & 15.60 \mathrm{~b} & 10.21 \mathrm{~b} \\ 35.75 \mathrm{a} & 35.45 \mathrm{a} & 33.60 \mathrm{c} & 10.14 \mathrm{c} & 10.29 \mathrm{c} & \\ 41.79 \mathrm{a} & 41.34 \mathrm{a} & & 12.97 \mathrm{a} & 12.16 \mathrm{a} & \end{array}$

Table 4: Effect of sewage water irrigation frequency on biomass production (on fresh and dry weight basis) of tomato and okra.

\section{Frequency of irrigation water}

Total plant biomass on fresh weight basis (g)

Frequent

Moderately frequent

Less frequent

Mean

Total plant biomass on dry weight basis $(\mathrm{g})$

Frequent

Moderately frequent

Less frequent

Mean

\begin{tabular}{llllll} 
& \multicolumn{2}{c}{ Tomato } & & \multicolumn{3}{c}{ Okra } \\
Year 2012 & Year 2013 & Mean & Year 2012 & Year 2013 & Mean \\
& & & & & \\
2885.8a & $2877.6 \mathrm{a}$ & $2881.7 \mathrm{a}$ & $514.92 \mathrm{a}$ & $521.39 \mathrm{a}$ & $518.15 \mathrm{~b}$ \\
$2599.7 \mathrm{~b}$ & $2556.4 \mathrm{~b}$ & $2578.0 \mathrm{~b}$ & $646.93 \mathrm{a}$ & $637.73 \mathrm{a}$ & $642.33 \mathrm{a}$ \\
$2297.5 \mathrm{c}$ & $2241.6 \mathrm{c}$ & $2269.6 \mathrm{c}$ & $440.27 \mathrm{a}$ & $443.03 \mathrm{a}$ & $441.65 \mathrm{c}$ \\
$2594.3 \mathrm{a}$ & $2558.5 \mathrm{a}$ & & $534.05 \mathrm{a}$ & $534.46 \mathrm{a}$ & \\
& & & & & \\
$318.76 \mathrm{a}$ & $319.14 \mathrm{a}$ & $318.95 \mathrm{a}$ & $90.27 \mathrm{a}$ & $90.91 \mathrm{a}$ & $90.59 \mathrm{~b}$ \\
$271.39 \mathrm{a}$ & $264.83 \mathrm{a}$ & $268.11 \mathrm{~b}$ & $125.71 \mathrm{a}$ & $123.31 \mathrm{a}$ & $124.51 \mathrm{a}$ \\
$230.35 \mathrm{a}$ & $224.96 \mathrm{a}$ & $227.66 \mathrm{c}$ & $76.42 \mathrm{a}$ & $72.98 \mathrm{a}$ & $74.70 \mathrm{c}$ \\
$273.50 \mathrm{a}$ & $269.64 \mathrm{a}$ & & $97.46 \mathrm{a}$ & $95.73 \mathrm{a}$ &
\end{tabular}

Treatment means sharing the different letter(s) in a group are significantly different at $p \geq 0.05$

\section{Heavy metals contents in tomato and okra roots}

Significantly greater metals contents $(\mathrm{Pb}, \mathrm{Ni}, \mathrm{Cu}, \mathrm{Cd}$ and $\mathrm{Cr}$ ) were recorded in tomato roots grown with frequent irrigation, followed by moderately frequent irrigation treatment. These two treatments were statistically at par. While, less frequent irrigation resulted in lower $\mathrm{Pb}, \mathrm{Ni}, \mathrm{Cu}, \mathrm{Cd}$ and $\mathrm{Cr}$ contents in roots, which was statistically similar to moderately frequent irrigation. Significantly higher Fe content was recorded in the roots of tomato plants under frequent irrigation treatment, while significantly lower $\mathrm{Fe}$ contents were observed with less frequent irrigation application (Table 7).

Significantly higher $\mathrm{Pb}$ and $\mathrm{Fe}$ contents were recorded in roots of okra plants grown with frequent and moderately frequent irrigation treatments. These two irrigation frequencies were statistically similar. Significantly lower $\mathrm{Pb}$ and $\mathrm{Fe}$ contents were noticed with less frequent irrigation application. Significantly greater $\mathrm{Ni}$ content was recorded in roots when plants were grown with frequent irrigation treatment, significantly lesser $\mathrm{Ni}$ content was recorded with less frequent irrigation treatment. Higher $\mathrm{Cu}, \mathrm{Cd}$ and 
$\mathrm{Cr}$ contents were recorded with frequent irrigation application, which was statistically at par with moderately frequent irrigation application. Lower $\mathrm{Cu}, \mathrm{Cd}$ and $\mathrm{Cr}$ contents were recorded with less frequent irrigation, which was also statistically similar with moderately frequent irrigation application.

Water shortage results in drought stress and poor growth and yields in crop plants. In the present study, significantly greater growth of tomato in terms plant height, relative leaf chlorophyll content and leaf area was recorded with frequent irrigation treatment (5 days interval), followed by moderately frequent (10 days interval) and less frequent irrigation treatment (15 days interval). The interaction between irrigation frequency treatments and years indicated that increased tomato growth (plant height and leaf area) was observed when plants were irrigated frequently during 2012 and 2013, while reduced growth was found with less frequent irrigation interval during both the years. The differences in growth parameters due to various irrigation frequency treatments during the years were possibly due to the availability of more water in frequent irrigation treatment and prevailing weather conditions of the area during the growing season. The current findings are also related with those of Abdel Mouty and Ali (2008), who found increased plant growth of eggplant when irrigated at 10 days irrigation interval than those irrigated with 21 days interval. Kirnak et al. (2001) also found that water shortage reduced plant height, leaf chlorophyll content and vegetative growth of eggplant when grown with different irrigation regimes (100, 80, 60 and 40\%). The number of eggplant leaves increased with increasing irrigation frequency (Byari and Al-Rabighi, 1996), being higher at shorter irrigation interval i.e., 6 days.

Table 5: Effect of serwage water irrigation frequency on heavy metals contents in tomato fruits and okra pods.

\section{Frequency of irrigation water}

$\mathrm{Pb}\left(\mathrm{mg} \mathrm{kg}^{-1}\right)$

Frequent

Moderately frequent

Less frequent

Mean

$\mathrm{Ni}\left(\mathrm{mg} \mathrm{kg}^{-1}\right)$

Frequent

Moderately frequent

Less frequent

Mean

$\mathrm{Cu}\left(\mathrm{mg} \mathrm{kg}^{-1}\right)$

Frequent

Moderately frequent

Less frequent

Mean

$\mathrm{Cd}\left(\mathrm{mg} \mathrm{kg}^{-1}\right)$

Frequent

Moderately frequent

Less frequent

Mean

$\mathrm{Fe}\left(\mathrm{mg} \mathrm{kg}^{-1}\right)$

Frequent

Moderately frequent

Less frequent

Mean

$\mathrm{Cr}\left(\mathrm{mg} \mathrm{kg}{ }^{-1}\right)$

Frequent

Moderately frequent

Less frequent

Mean

\section{Tomato fruits}

Year 2013

$8.200 a$

$7.200 \mathrm{a}$

$5.567 \mathrm{a}$

$6.988 \mathrm{a}$

$8.933 a$

$7.400 \mathrm{a}$

$6.100 \mathrm{a}$

7.477a

$12.800 \mathrm{a}$

10.600a

$8.700 \mathrm{a}$

$10.700 \mathrm{a}$

$13.167 \mathrm{a}$

11.13a

$9.900 \mathrm{a}$

$11.067 \mathrm{a}$

$9.633 a$

$7.700 \mathrm{a}$

$6.500 \mathrm{a}$

$7.944 \mathrm{a}$

$2.566 \mathrm{a}$

$2.166 \mathrm{a}$

$1.700 \mathrm{a}$

$2.144 \mathrm{a}$

$137.00 \mathrm{a}$

$9.966 \mathrm{a}$

7.666.a

$6.600 \mathrm{a}$

$8.077 \mathrm{a}$

$116.43 \mathrm{a}$

$80.23 \mathrm{a}$

$111.22 \mathrm{a}$

$0.500 \mathrm{a}$

$0.333 \mathrm{a}$

$0.166 \mathrm{a}$

$0.333 \mathrm{a}$
$2.700 \mathrm{a}$

$2.233 \mathrm{a}$

$1.800 \mathrm{a}$

$2.244 \mathrm{a}$

$139.57 \mathrm{a}$

$117.67 \mathrm{a}$

$92.80 \mathrm{a}$

$116.68 \mathrm{a}$

$0.603 a$

$0.366 \mathrm{a}$

$0.233 a$

$0.401 \mathrm{a}$
Mean

$8.566 a$

7.300ab

$5.833 \mathrm{~b}$

12.983a

$10.867 \mathrm{ab}$

$8.800 \mathrm{~b}$

$9.800 \mathrm{a}$

$7.683 \mathrm{~b}$

$6.550 \mathrm{~b}$

2.633a

$2.200 \mathrm{~b}$

$1.750 \mathrm{c}$

$138.28 \mathrm{a}$

$117.05 b$

$86.52 \mathrm{c}$

$0.551 \mathrm{a}$

$0.350 \mathrm{ab}$

$0.200 \mathrm{~b}$

\section{Year 2012}

$3.300 \mathrm{a}$

$2.766 \mathrm{a}$

$2.4333 a$

$2.833 a$

$8.800 a$

$8.200 \mathrm{a}$

$7.400 \mathrm{a}$

$8.133 a$

$10.800 a$

$9.400 \mathrm{a}$

$8.200 \mathrm{a}$

$9.467 \mathrm{a}$

$0.083 a$

$0.053 a$

$0.033 \mathrm{a}$

$0.056 \mathrm{a}$

203.93a

143.60a

128.07a

$158.53 \mathrm{a}$

$0.900 \mathrm{a}$

$0.500 \mathrm{a}$

$0.200 \mathrm{a}$

$0.533 \mathrm{a}$
Okra pods

Year 2013 Mean

$3.500 \mathrm{a}$

$3.400 \mathrm{a}$

$3.100 \mathrm{a}$

$2.933 b$

$2.600 \mathrm{a}$

$2.516 \mathrm{~b}$

$3.066 \mathrm{a}$

$9.200 \mathrm{a}$

$9.000 \mathrm{a}$

$8.500 \mathrm{a}$

$8.350 \mathrm{ab}$

$7.800 \mathrm{a}$

$7.600 \mathrm{~b}$

$8.500 \mathrm{a}$

$11.233 \mathrm{a}$

$11.017 \mathrm{a}$

$10.500 \mathrm{a}$

$9.950 \mathrm{a}$

$9.033 \mathrm{a}$

$8.617 \mathrm{~b}$

$10.256 \mathrm{a}$

$0.333 \mathrm{a}$

$0.208 \mathrm{a}$

$0.059 \mathrm{a}$

$0.056 \mathrm{ab}$

$0.040 \mathrm{a}$

$0.036 \mathrm{~b}$

$0.144 \mathrm{a}$

$210.47 \mathrm{a}$

207.20a

$162.60 \mathrm{a}$

$153.10 \mathrm{~b}$

$138.10 \mathrm{a}$

$133.08 \mathrm{c}$

$170.39 \mathrm{a}$

$1.333 \mathrm{a}$

$1.116 \mathrm{a}$

$0.633 \mathrm{a}$

$0.566 \mathrm{~b}$

$0.366 \mathrm{a}$

$0.283 \mathrm{~b}$

Treatment means sharing the different letter(s) in a group are significantly different at $p \geq 0.05$ 
Table 6: Effect of serwage water irrigation frequency on heavy metals contents in tomato and okra leaves.

\section{Frequency of irrigation water}

$\mathrm{Pb}\left(\mathrm{mg} \mathrm{kg}^{-1}\right)$

Frequent

Moderately frequent

Less frequent

Mean

$\mathrm{Ni}\left(\mathrm{mg} \mathrm{kg}^{-1}\right)$

Frequent

Moderately frequent

Less frequent

Mean

$\mathrm{Cu}\left(\mathrm{mg} \mathrm{kg}^{-1}\right)$

Frequent

Moderately frequent

Less frequent

Mean

$\mathrm{Cd}\left(\mathrm{mg} \mathrm{kg}^{-1}\right)$

Frequent

Moderately frequent

Less frequent

Mean

$\mathrm{Fe}\left(\mathrm{mg} \mathrm{kg}^{-1}\right)$

Frequent

Moderately frequent

Less frequent

Mean

$\mathrm{Cr}\left(\mathrm{mg} \mathrm{kg}{ }^{-1}\right)$

Frequent

Moderately frequent

Less frequent

Mean
Tomato fruits

Year 2013

Year 2012

$9.400 \mathrm{a}$

$7.800 \mathrm{a}$

$5.200 \mathrm{a}$

7.466

$16.600 \mathrm{a}$

$15.300 \mathrm{a}$

$13.533 \mathrm{a}$

$15.144 \mathrm{a}$

$11.633 a$

$9.767 \mathrm{a}$

$9.200 \mathrm{a}$

$10.200 \mathrm{a}$

$2.866 \mathrm{a}$

$2.466 \mathrm{a}$

$1.866 \mathrm{a}$

$2.400 \mathrm{a}$

249.37a

236.70a

$155.87 \mathrm{a}$

213.98a

$1.600 \mathrm{a}$

$1.000 \mathrm{a}$

$0.701 \mathrm{a}$

$1.100 \mathrm{a}$

7.988a

$15.633 \mathrm{a}$

13.633a

$15.322 \mathrm{a}$

12.267a

$9.967 \mathrm{a}$

$9.300 \mathrm{a}$

10.511a

$3.166 \mathrm{a}$

2.333a

$2.766 \mathrm{a}$

240.20a

157.13a

219.68a

$1.900 \mathrm{a}$

$1.166 \mathrm{a}$

$0.700 \mathrm{a}$

$1.255 \mathrm{a}$

$\begin{array}{ll}9.966 \mathrm{a} & 9.683 \mathrm{a} \\ 7.866 \mathrm{a} & 7.833 \mathrm{~b} \\ 6.133 \mathrm{a} & 5.666 \mathrm{c}\end{array}$

Mean

Year 2012

Okra pods

Year 2013

Mean

$4.800 \mathrm{a}$

$5.300 \mathrm{a}$

$5.050 \mathrm{a}$

$4.033 \mathrm{a}$

$4.200 \mathrm{a}$

4.116ab

3.601a

$3.600 \mathrm{a}$

$3.600 \mathrm{~b}$

$4.200 \mathrm{a}$

$4.311 \mathrm{a}$

$16.700 \mathrm{a} \quad 16.650 \mathrm{a}$

$10.533 a$

$11.500 \mathrm{a}$

$11.017 \mathrm{a}$

$15.467 \mathrm{a}$

$9.633 a$

$9.700 \mathrm{a}$

9.667ab

$13.583 b$

$8.833 \mathrm{a}$

$8.840 \mathrm{a}$

$8.833 \mathrm{~b}$

$9.667 \mathrm{a}$

$10.011 \mathrm{a}$

$11.950 \mathrm{a}$

$13.933 \mathrm{a}$

14.667a

$14.050 \mathrm{a}$

$9.867 \mathrm{~b}$

$12.433 \mathrm{a}$

$12.967 \mathrm{a}$

$12.700 \mathrm{ab}$

$9.250 \mathrm{~b}$

$11.367 \mathrm{a}$

$11.267 \mathrm{a}$

$11.317 \mathrm{~b}$

$12.578 \mathrm{a}$

$12.800 \mathrm{a}$

$3.016 \mathrm{a}$

$0.800 \mathrm{a}$

$0.966 \mathrm{a}$

$0.883 \mathrm{a}$

2.633ab

$0.433 \mathrm{a}$

$0.700 \mathrm{a}$

$0.566 \mathrm{~b}$

$2.100 \mathrm{~b}$

$0.300 \mathrm{a}$

$0.366 \mathrm{a}$

$0.333 \mathrm{~b}$

$0.511 \mathrm{a}$

$0.677 \mathrm{a}$

$261.70 \mathrm{a} \quad 255.53 \mathrm{a}$

259.63a

$262.90 \mathrm{a}$

261.27a

$238.45 \mathrm{~b}$

$244.57 \mathrm{a}$

245.43a

245.00a

$156.50 \mathrm{c}$

214.00a

214.63a

214.32b

239.40a

240.99 a

$1.750 \mathrm{a}$

$2.200 \mathrm{a}$

$2.300 \mathrm{a}$

$2.250 \mathrm{a}$

$1.083 \mathrm{~b}$

$1.800 \mathrm{a}$

$1.866 \mathrm{a}$

1.833ab

$0.700 \mathrm{~b}$

$1.500 \mathrm{a}$

$1.400 \mathrm{a}$

$1.450 \mathrm{~b}$

$1.833 \mathrm{a}$

$1.8553 \mathrm{a}$

Treatment means sharing the different letter(s) in a group are significantly different at $p \geq 0.05$

In the present study, moderately frequent irrigation (10 days interval) gave significantly greater plant height and leaf area of okra than frequent (5 days interval) and less frequent irrigation treatments (15 days interval). This study supports the previous findings of Khalil (2004), who found increased growth rate in terms of plant height and number of leaves per plant when the plants were grown with 10 days' irrigation interval. Abbas (2007) found that irrigation at every 8 days' interval gave significantly taller okra plants. The variations in the present and previous results could be due to soil type and environmental differences. The increased growth of summer vegetables by the application of frequent or moderately frequent irrigations with sewage water was possibly due to that this water not only provides moisture but also contains nutrients required for proper growth and development.

In the present study, frequent irrigation application significantly increased the number of fruits per plant, fruit length, fruit diameter, fresh and dry weight of individual fruit and yield per hectare in tomato compared with moderately frequent and less frequent irrigation treatments. The larger size of tomato fruits was probably due to proper supply of water at particular development stage such as reproductive phase, which needs more supply of water and nutrients for the production of an appreciable size of fruits. The yield of tomato significantly increased with increasing 
the frequency of sewage water (Phene et al., 1986). Gudugi et al. (2012) reported that irrigation interval at 4 days resulted in a higher number of tomato fruits and greater yield than with 8 days interval during 2010-2011, possibly associated with conditions of low humidity and high temperature, which could quickly dry the soil. Less availability of irrigation water negatively affects the hormonal balance, plant growth, development, and nutrients translocation (Salisbury and Ross, 1992). Bafeel and Moftah (2008) concluded that reduced vegetative growth and negative effect on yield and its components in eggplants could be related to drought stress. Similar results were also achieved for tomato by Mahmood (2008).

The present study indicated that the irrigation regimes used have a significant effect on yield and yield components of okra. This difference in yield was possibly due to excessive and low irrigation application to okra crop (Al-Harbi et al., 2008). The decreased yield was also observed in water-stressed okra plants (Sankar et al., 2008). The current findings are connected with the conclusions of Khalil (2004), who found significantly greater pod length, pod weight and yield of okra under 10 days irrigation interval. This difference in the results was due to different soil types and environmental conditions of the growing areas. The increased yield in the present study was also possibly due to the application of sewage water, as this water is enriched with macro and micronutrients and organic matter, which ultimately increased yields of the studied vegetables.

Table 7: Effect of sewage water irrigation frequency on heavy metal contents in tomato and okra roots.

\section{Frequency of irrigation water}

\section{$\mathrm{Pb}\left(\mathrm{mg} \mathrm{kg}^{-1}\right)$}

Frequent

Moderately frequent

Less frequent

Mean

$\mathrm{Ni}\left(\mathrm{mg} \mathrm{kg}^{-1}\right)$

Frequent

Moderately frequent

Less frequent

Mean

$\mathrm{Cu}\left(\mathrm{mg} \mathrm{kg}^{-1}\right)$

Frequent

Moderately frequent

Less frequent

Mean

$\mathrm{Cd}\left(\mathrm{mg} \mathrm{kg}^{-1}\right)$

Frequent

Moderately frequent

Less frequent

Mean

Fe $\left(\mathrm{mg} \mathrm{kg}^{-1}\right)$

Frequent

Moderately frequent

Less frequent

Mean

$\mathrm{Cr}\left(\mathrm{mg} \mathrm{kg}^{-1}\right)$

Frequent

Moderately frequent

Less frequent

Mean
Tomato fruits

$\begin{array}{llllll}\text { Year 2012 } & \text { Year 2013 } & \text { Mean } & \text { Year 2012 } & \text { Year 2013 } & \text { Mean } \\ & & & & & \\ 12.966 \mathrm{a} & 13.200 \mathrm{a} & 13.083 \mathrm{a} & 7.533 \mathrm{a} & 8.100 \mathrm{a} & 7.816 \mathrm{a} \\ 9.500 \mathrm{a} & 11.500 \mathrm{a} & 10.500 \mathrm{ab} & 6.700 \mathrm{a} & 7.200 \mathrm{a} & 6.950 \mathrm{a} \\ 7.533 \mathrm{a} & 9.600 \mathrm{a} & 8.567 \mathrm{~b} & 5.400 \mathrm{a} & 6.300 \mathrm{a} & 5.850 \mathrm{~b} \\ 10.00 \mathrm{a} & 11.43 \mathrm{a} & & 6.544 \mathrm{a} & 7.200 \mathrm{a} & \end{array}$

\section{$19.633 \mathrm{a}$}

$17.633 \mathrm{a}$

$23.167 \mathrm{a}$

$19.400 \mathrm{a}$

$15.700 \mathrm{a}$

17.656a

$17.800 \mathrm{a}$

$20.122 \mathrm{a}$

$16.600 \mathrm{a}$

14.733a

$12.600 \mathrm{a}$

$14.644 a$

$3.800 \mathrm{a}$

$3.400 \mathrm{a}$

$2.733 \mathrm{a}$

3.311a

$289.27 \mathrm{a}$

252.47a

$172.43 \mathrm{a}$

$238.06 a$

$2.433 \mathrm{a}$

$1.800 \mathrm{a}$

$1.200 \mathrm{a}$

$1.811 \mathrm{a}$
$17.500 \mathrm{a}$

$15.567 \mathrm{a}$

$13.700 \mathrm{a}$

$15.589 \mathrm{a}$

4.200a

$3.700 \mathrm{a}$

$3.200 \mathrm{a}$

$3.700 \mathrm{a}$

313.63a

266.13a

196.00a

258.59 a

$2.200 \mathrm{a}$

$1.900 \mathrm{a}$

$2.266 \mathrm{a}$ 2.700a
$21.400 \mathrm{a}$

18.517ab

$13.500 \mathrm{a}$

$11.700 \mathrm{a}$

$10.067 \mathrm{a}$

$11.756 a$

$13.600 \mathrm{a}$

$12.500 \mathrm{a}$

$10.200 \mathrm{a}$

$12.100 \mathrm{a}$

$17.050 \mathrm{a}$

$16.400 \mathrm{a}$

$15.633 \mathrm{a}$

$15.533 \mathrm{a}$

$15.300 \mathrm{a}$

$13.500 \mathrm{a}$

$13.967 \mathrm{a}$

$15.067 \mathrm{a}$

$15.044 \mathrm{a}$

$4.000 \mathrm{a}$

$1.333 \mathrm{a}$

$1.416 \mathrm{a}$

$1.375 \mathrm{a}$

$3.550 \mathrm{ab}$

$0.700 \mathrm{a}$

$2.966 \mathrm{~b}$

$0.300 \mathrm{a}$

$0.744 \mathrm{a}$

$1.100 \mathrm{a}$

$0.900 \mathrm{ab}$

$0.6300 \mathrm{a}$

$0.466 \mathrm{~b}$

$1.050 \mathrm{a}$

$301.45 \mathrm{a}$

$310.50 \mathrm{a}$

$327.53 a$

$311.43 a$

$259.30 \mathrm{~b}$

295.33

$304.57 \mathrm{a}$

307.53a

$184.22 \mathrm{c}$

$271.77 \mathrm{a}$

$270.47 \mathrm{a}$

$71.12 \mathrm{~b}$

$290.56 \mathrm{a}$

$302.83 \mathrm{a}$

$\begin{array}{ll}2.566 \mathrm{a} & 2.600 \mathrm{a} \\ 2.000 \mathrm{ab} & 2.333 \mathrm{a} \\ 1.550 \mathrm{~b} & 1.866 \mathrm{a} \\ & 2.266 \mathrm{a}\end{array}$

$2.933 a$

$2.766 \mathrm{a}$

$2.533 \mathrm{a}$

$2.433 \mathrm{ab}$

$2.200 \mathrm{a}$

$2.033 \mathrm{~b}$
$15.150 \mathrm{ab}$

Treatment means sharing the different letter(s) in a group are significantly different at $p \geq 0.05$ 
Total fresh and dry plant tomato plant biomass was significantly greater with 5 days sewage irrigation frequency, followed by 10 days interval and the minimum was recorded with 15 days irrigation interval. Reduced dry weight of shoot and leaves of tomato results usually due to insufficient soil moisture (Singandhupe et al., 2003).Various plant physiological processes might be hampered due to inadequate availability of soil water. Water stress tends to decrease root growth of the tomato plant, which leads to reduced shoot dry weight because the development of roots and shoots are closely related (Ismail et al., 2008). In another study, total tomato plant biomass was decreased with water stress (Birhanu and Tilahun, 2010).

In the present study, significantly increased fresh and dry total plant biomass were obtained in okra plants grown with moderately frequent irrigation (10 days interval) than frequent ( 5 days interval) and less frequent irrigation treatment (15 days interval). Reduction in biomass of okra plant due to water stress has already been reported by Sankar et al. (2008).

Significantly greater $\mathrm{Pb}, \mathrm{Cr}, \mathrm{Ni}, \mathrm{Fe}, \mathrm{Cu}$ and $\mathrm{Cd}$ substances were noted in the cultivated tomato fruits and okra pods when plants were irrigated frequently (5 days interval), while less frequent irrigation treatment (15 days interval) resulted in lesser accumulation of these heavy metals. All the fruit and pod samples of tomato and okra plants under all three irrigation frequency treatments exhibited $\mathrm{Pb}$ and $\mathrm{Cd}$ contents exceeding above the higher approved limits set by Asaolu (1995) and WHO (1996). Ni contents in fruits of tomato plants irrigated with frequent and moderately frequent irrigation treatments were above the maximum permissible limits, and these contents were below the permissible limits when less frequent irrigation was applied. Whereas $\mathrm{Cu}, \mathrm{Fe}$ and $\mathrm{Cr}$ contents in fruits of tomato plants irrigated with all the three irrigation frequency treatments were below the maximum permissible limits. The $\mathrm{Ni}$ and $\mathrm{Cr}$ contents in okra pods were found below the safer limits when grown with all the three irrigation frequency treatments. The pods contained $\mathrm{Cu}$ contents above the permissible limits when sewage water was applied frequently, while moderately and less frequent irrigation treatments resulted in $\mathrm{Cu}$ contents below the recommended levels. Frequent and moderately frequent sewage water applications showed higher Fe contents in pods above the permissible limits, while less frequent sewage water application showed lower content in pods lower the safe limits, recognized by Asaolu (1995) and WHO (1996).

Application of sewage water continuously pollutes the soils with heavy metals. Soils act as filters of poisonous compounds, possibly absorbing and retaining heavy metals from sewage water. When soils' capacity to retain heavy metals, contents is decreased due to loading of these contaminants continuously or soil $\mathrm{pH}$ changes, then soils can discharge these toxic metals into water or soil solution and make available for plants uptake. The results of the present study indicated that as the frequency of sewage irrigation application was increased, there was more accumulation of heavy metals in plant parts. It was possibly due to more buildup of heavy metals in soil because of indiscriminate use of sewage water which ultimately contaminated the crop plants grown on those soils. It was observed that as the duration of sewage irrigation interval increased, there was less accumulation of heavy metals in plant parts. It is because the heavy metals contents were increased with frequent irrigation treatment and decreased when grown with moderately and less frequent irrigation applications in the present investigation.

Tomato and okra leaves and roots showed all the heavy metals contents as affected by the frequency of irrigation treatments in order: frequent $>$ moderately frequent > less frequent sewage water application. With the decrease in duration of sewage irrigation application, the metals tended to accumulate in the soil, and their availability in soil solution and uptake resulted in greater metal content in plant parts. Therefore, the frequent irrigation water increased the heavy metals contents in tomato and okra plant parts as compared to moderately frequent irrigation application, while less frequent irrigation application resulted in lower metals contents in plant parts. Thus, the frequent use of sewage water for irrigation has a greater ability to pollute the soil, which in turn results in uptake of metals by crops.

\section{Acknowledgement}

The data presented in this manuscript is a part of Ph.D. thesis research conducted at the Department of Horticulture, Bahauddin Zakariya University, Multan. The authors are grateful to the Higher Education Commission for financial assistance to 
conduct the research under the Indigenous $\mathrm{PhD}$ Fellowship Program.

\section{Novelty Statement}

Novelty of the current research highlights the malpractices performed in olericulture by farming community by the frequent use of sewage water application in an increased amount which results in the accumulation of $\mathrm{Fe}, \mathrm{Cu}, \mathrm{Pb}, \mathrm{Cr}, \mathrm{Ni}$ and $\mathrm{Cd}$ in higher amounts in okra and tomato in pods and fruits respectively. Resultantly these commodities are used as food and a serious hazard for human health.

\section{Author's Contribution}

The idea was conceived by Safina Naz and Muhammad Akbar Anjum. The experiments were performed by Safina Naz, Sajid Ali, Hassan Sardar and Sakeena Tul Ain Haider. Statistical analysis was performed by Syed Atif Hasan Naqvi and Zehri Khan. The overall management of the article was performed by Muhammad Asif Zulfiqar, Sibghat Ullah, Hajra Azeem and Shah Pasand.

\section{Conflict of interest}

The authors have declared no conflict of interest.

\section{References}

Abbas, J.A., 2007. Effect of potassium fertilization and irrigation intervals on growth and yield of eggplant Solanum melogena L. Jordan J. Agric. Sci., 3: 350-361.

Abdel-Mouty, M.M. and A.H. Ali. 2008. Combined effect of irrigation intervals and foliar application of some antitranspirants on eggplant growth, fruits yield and its physical and chemical properties. Res. J. Agric. Biol. Sci., 4: 416-423.

Al-Harbi, A.R., A.M. AI-Orman and I.F.I. E1Adgham, 2008. Effect of drip irrigation levels and emitters depth on okra (Abelmoschus esculentus) growth. J. Appl. Sci., 8: 2764- 2769. https://doi.org/10.3923/jas.2008.2764.2769

Aparicio, J., L. Santos and E. Alonso. 2007. Simultaneous sonication-assisted extraction, and determination by gas chromatography-mass spectrometry, of di-(2-ethylhexyl) phthalate, nonylphenol, nonylphenol ethoxylates and polychlorinated biphenyls in sludge from waste water treatment plants. Anal. Chim. Acta, 584: 455-461. https://doi.org/10.1016/j. aca.2006.11.039

Asaolu, S.S., 1995. Lead content of vegetable and tomatoes at Erekesan Market, Ado-Ekiti. Pak. J. Sci. Indus. Res., 38: 399-401.

Bafeel, S.O. and A.E. Moftah. 2008. Physiological response of eggplants grown under different irrigation regimes to antitransplant treatments. Saudi J. Biol. Sci., 15: 259-267.

Birhanu, K. and K. Tilahun. 2010. Fruit yield and quality of drip-irrigated tomato under deficit irrigation. Afric. J. Food Agric. Nutr. Dev., 10: 2139-2151. https://doi.org/10.4314/ajfand. v10i2.53356

Byari, S.H. and S.M.S. Al-Rabighi. 1996. Yield and growth responses of eggplant cultivars to water deficit. Egypt. J. Hortic., 23: 89-100.

Farooq, M., F. Anwar and U. Rashid. 2008. Appraisal of heavy metal contents in different vegetables grown in the vicinity of an industrial area. Pak. J. Bot., 40: 2099-2106.

Gudugi, I.A.S., A.J. Odofin, M.K.A. Adeboye and J.A. Oladiran. 2012. Agronomic characteristics of tomato as influenced by irrigation and mulching. Adv. Appl. Sci. Res., 3: 2539-2543.

Ismail, S.M., K. Ozawa and N.A. Khondaker. 2008. Influence of single and multiple water application timings on yield and water use efficiency in tomato (var. First power). Agric. Water Manage., 95: 116-122. https://doi. org/10.1016/j.agwat.2007.09.006

Khalil, H.A.Y., 2004. Improvement of some okra (Abelmoschus esculentus L. Moench) cultivars under drought conditions. Ph.D. thesis, Department of Horticulture, Faculty of Agriculture, Assiut University, Assiut Egypt.

Khan, M.J., M.T. Jan, N.U. Farhatullah, M.A. Khan, S. Perveen, S. Alam and A.U. Jan. 2011. The effect of using waste water for tomato. Pak. J. Bot., 43: 1033-1044.

Kirnak, H., C. Kaya, I. Tas and D. Higgs. 2001. The influence of water deficit on vegetative growth, physiology, fruit yield and quality in eggplants. Bulgarian J. Plant Physiol., 27: 34-46.

Mahmood, T., 2008. Path coefficient analysis of yield component in tomato (Lycopersicon esculentum). Pak. J. Bot., 40: 627-635.

Mani, H., S.A. Rahman and B.A. Babaji. 2007. Yields and economic returns of tomato at different irrigation intervals, spacing and 
planting positions at Samaru in Northern

Nigeria. J. Sustainable Trop. Agric. Res., 21: 4952.

Moniruzzaman, M., S.M. Faisal, M.A.R. Sarkar, M.I. Hossain, M.A. Ali and M.A.H. Talukder. 2007. Effects of irrigation and different mulches on yield of profitability of cauliflower. Asian J. Plant Sci., 6: 338-343. https://doi.org/10.3923/ ajps.2007.338.343

Naveed, A., A.A. Khan and I.A. Khan. 2009. Generation mean analysis of water stress tolerance in okra (Abelmoschous esculentus L.). Pak. J. Bot., 41: 195-205.

Phene, C.J., K.R. Davis, R.B. Hutmacher and R.L. McCormick. 1986. Advantages of subsurface irrigation for processing tomatoes. II Int. Symp. Proc. Tomatoes. $11^{\text {th }}$ August, 1986. XXII IHC 200. pp. 101-114. https://doi.org/10.17660/ ActaHortic.1987.200.9

Rehman, A.B., 2007. Pharmacological studies on traditional medicine (Cyperus rotundus) used in Pakistan. University of Karachi.

Ramalan, A.A. and C.U. Nwokeocha. 2000. Effects of furrow irrigation methods, mulching and soil water suction on the growth, yield and water use efficiency of tomato in the Nigerian savanna. Agric. Water Manage., 45: 317-330. https:// doi.org/10.1016/S0378-3774(99)00104-3

Salisbury, F.B. and C.W. Ross. 1992. Environmental physiology: In: Plant physiology, $4^{\text {th }}$ Ed.
Wadsworth Publishing Company, Belmont CA, USA. pp. 549-600.

Sankar, B., C.A. Jaleel, P. Manivannan, A. Kishorekumar, R. Somasundaram and R. Panneerselvam. 2008. Relative efficacy of water use in five varieties of Abelmoschus esculentus (L.) Moench under water-limited conditions. Colloids Surfaces B: Biointerfaces, 62: 125-129. https://doi.org/10.1016/j.colsurfb.2007.09.025

Singandhupe, R.B., G.G.S.N. Rao, N.G. Patil and P.S. Brahmanand. 2003. Fertigation studies and irrigation scheduling in drip irrigation system in tomato crop (Lycopersicon esculentum L.). Eur. J. Agron., 19: 327-340. https://doi.org/10.1016/ S1161-0301(02)00077-1

WHO, 1996. Guidelines for drinking water quality, health criteria and supporting information. 94/9960- Mastercom/ Wiener Verlag-800, Australia.

Yadav, S.K. and B.S. Dhankhar. 1999. Performance of Varsha Uphar cultivar of okra (Abelmoschus esculentus (L.) Moench) as affected by the sowing dates and plant geometry. Veg. Sci., 26: 180-182.

Yazgan, S., S. Ayas, C. Demirtas, H. Buyukcangaz and B.N. Candogan. 2008. Deficit irrigation effects on lettuce (Lactuca sativa var. Olenka) yield in unheated greenhouse condition. J. Food Agric. Environ., 6: 357-361. 